\title{
Ivermectin Treatment Coverage Validation in Two Onchocerciasis Endemic Districts in Ethiopia: A Community-Based Cross-Sectional Study, 2019
}

This article was published in the following Dove Press journal: Journal of Multidisciplinary Healthcare

\author{
Chuchu Churko (D) \\ Manaye Yihune (D) $^{2}$ \\ Abinet Teshome ${ }^{3}$ \\ Yilma Chisha ${ }^{2}$ \\ Birhanu Getachew ${ }^{4}$ \\ Markos Sleshi ${ }^{4}$ \\ Mekuria Asnakew Asfaw (D) \\ Tamiru Shibiru ${ }^{5}$ \\ Nebiyu Negussu Ayele ${ }^{6}$ \\ Fikre Seife ${ }^{6}$ \\ Zerihun Zerdo' \\ Alemayehu Bekele Kassahun (D) ' \\ 'Collaborative Research and Training \\ Center for Neglected Tropical Diseases, \\ College of Medicine and Health Sciences, \\ Arba Minch University, Arba Minch, \\ Ethiopia; ${ }^{2}$ Department of Public Health, \\ College of Medicine and Health Sciences, \\ Arba Minch University, Arba Minch, \\ Ethiopia; ${ }^{3}$ Department of Biomedical \\ Sciences, College of Medicine and Health \\ Sciences, Arba Minch University, Arba \\ Minch, Ethiopia; ${ }^{4}$ Ethiopia Public Health \\ Institute, Addis Ababa, Ethiopia; ${ }^{5}$ School \\ of Medicine, College of Medicine and \\ Health Sciences, Arba Minch University, \\ Arba Minch, Ethiopia; ${ }^{6}$ Neglected \\ Tropical Diseases, Federal Ministry of \\ Health, Addis Ababa, Ethiopia
}

Correspondence: Chuchu Churko Email churkochuchu2005@gmail.com
Background: Onchocerciasis is the second leading cause of blindness globally next to trachoma, thus eliminating the infection is an important health priority. It is estimated that 15.7 million people are at risk of infection in different parts of Ethiopia. Mass drug administration with ivermectin at community and school level is the basis for control and elimination of onchocerciasis. This study was aimed at validating onchocerciasis treatment coverage in the selected districts of Ethiopia.

Methods: A community-based cross-sectional study was employed in Itang special and Wombera districts of Ethiopia, from April 1 to 30, 2019 G.C. We used a coverage validation survey builder tool to compute sample size. Individuals aged five years old and above were eligible population. Data were entered into Microsoft Excel and exported to STATA 14 for cleaning and analyses. A chi-square test was used to note statistical association of the outcome variables with independent variables.

Main Findings: A total of 3765 individuals were interviewed. Of these, 3244 were offered onchocerciasis treatment. The overall treatment coverage of onchocerciasis in the two selected districts of Ethiopia was $85.9 \%$ of the eligible population $(3235 / 3765)(95 \% \mathrm{CI}$, $84.8 \%, 87 \%$ ). There was significant difference between the two districts in terms of ivermectin offering $\left(X^{2}=70.467, P<0.001\right)$. School attendance was also significantly associated with treatment offering and swallowing status $\left(X^{2}=77.29, P<0.001\right.$; and $X^{2}=30.581$, $P<0.001)$. The main reported reasons for not being offered ivermectin were "being absent" (40.86\%) and "not knowing about the mass drug administration" (MDA) (25.29\%).

Conclusion: In conclusion, the treatment coverage of onchocerciasis in this survey was higher than minimum national desired therapeutic coverage. Treatment coverage in Wombera was higher than Itang special district. In addition, children who attended school had a higher chance of swallowing the drug.

Keywords: ivermectin, onchocerciasis, coverage survey, mass drug administration

\section{Background}

Onchocerciasis - or "river blindness" - is a parasitic disease caused by the filarial nematode called Onchocerca volvulus. The life cycle of the parasite depends on the bites of black flies of the genus Simulium that breed along streams and rivers in areas where there is fast-flowing water. About 205 million people live in areas that are known to be endemic for onchocerciasis. Many of these people live in areas where there is little risk for onchocerciasis-related blindness or skin disease, as long as mass drug administration (MDA) with ivermectin (IVM) continues. ${ }^{1}$ 
A study conducted in 2017 by the Global Burden of Disease Study estimated that there were 20.9 million prevalent $O$. volvulus infections worldwide; 14.6 million and 1.15 million of the infected people had skin disease and vision loss, respectively. More than $99 \%$ of infected people live in 31 African countries. The disease also exists in some foci in Latin America and Yemen. ${ }^{2}$ The infection causes severe and upsetting skin disease and is related directly with higher death rates. ${ }^{3,4}$ According to the Global Burden of Disease report onchocerciasis was accountable for 34,600 disability-adjusted life-years (DALYs) lost. ${ }^{5}$ It finally leads to reduced agricultural productivity and largely contributes to poverty over generations. ${ }^{6}$

Almost all (99\%) of all current cases are found in SubSaharan Africa (SSA) where it has been a serious public health problem and has hindered socioeconomic development in endemic areas. ${ }^{7,8}$ The Onchocerciasis Control Program (OCP) and the African Programme for Onchocerciasis Control (APOC) have reduced the impact of disease as it is no longer a public health problem in most endemic areas through the use of communitydirected treatment with ivermectin (CDTI). ${ }^{9}$

In the African region, the number of people who received onchocerciasis treatment became increasing from time to time. This assisted the trend of increasing coverage of onchocerciasis treatment in 2018, with 15 countries reporting $100 \%$ geographical coverage. In an area where a total of 216.4 million people were at risk, coverage of onchocerciasis treatment was $69.9 \%$. Three countries Ethiopia, Nigeria and Uganda - had stopped MDA in at least 1 subnational area, after meeting the criteria in the 2016 WHO guide-lines, and started post-treatment survey. ${ }^{10}$ In Ethiopia, an epidemiological mapping survey conducted in 2011 reported that Amhara, Oromia, Southern Nations, Nationalities and Peoples' Region (SNNPR), Gambella and Benishangul-Gumuz regions are hyper- and mesoendemic for onchocerciasis. It is estimated that more than 15 million people are at risk of infection in 179 districts in the aforementioned regions. ${ }^{8}$ A study conducted in Oromia region Jimma and West Welega zones of Ethiopia reported the prevalence of onchocerciasis as $22.5 \%$ and $74.8 \%$, respectively. ${ }^{11,12}$ The central highlands and arid lowlands of Ethiopia are generally thought to be free of onchocerciasis. This is likely due to the highland's low temperature and the lowland's slow-flow rivers, the dry weather and a lack of suitable vegetation, which are unsuitable environments for both the vector and the parasite. ${ }^{4}$
Ivermectin (Mectizan ${ }^{\circledR}$ ) is an oral microfilaricidal drug that is safe and effective for mass treatment of onchocerciasis and is provided free to endemic countries. Interrupting transmission would allow countries to protect the gains made by many years of effective MDA with ivermectin and to stop MDA permanently, freeing the resources for other health priorities. It would be a meaningful contribution to Sustainable Development Goal (SDG) 3.3, part of which calls for ending the epidemics of AIDS, tuberculosis, malaria and neglected tropical diseases (NTDs) by 2030. ${ }^{13,14}$

In line with WHO strategy, the Ethiopian government introduced a control and elimination program for onchocerciasis and other NTDs, aiming to remove them as a public health problem by $2020 .^{13,14}$ The country has successfully scaled up interventions and achieved 100\% geographic coverage in all known endemic districts. The main strategy for interrupting the disease is MDA delivered two times per year. The treatment coverage for the last five years has been maintained at more than $80 \%$. In spite of many years of ivermectin MDA the transmission of onchocerciasis in many districts continued unchanged. To achieve the 2020 goal, and or beyond, sustained high geographic and therapeutic coverage is required which is validated by coverage surveys. ${ }^{15}$ To achieve this goal mass drug administration for onchocerciasis with ivermectin has been given for individuals living in endemic areas of Ethiopia prior to the two months of this survey. Although we had routine coverage reports in the health system, these reports are subject to errors and their accuracy is in doubt. Therefore, the aim of this survey was to assess validated treatment coverage of ivermectin against onchocerciasis and to determine treatment coverage disaggregated by sex, age and school attendance status.

\section{Materials and Methods Study Setting}

Benishangul-Gumuz and Gambella regional states of Ethiopia were selected for this study from a sentinel site list provided by Federal Ministry of Health $(\mathrm{FMoH})$. These regional states were purposively selected due to endemicity of onchocerciasis and based on doubt on routine reports of treatment coverage.

From each region one district (Itang special from Gambella and Wombera from Benishangul-Gumuz) was randomly selected from districts which distributed the treatment. These two districts conducted mass drug 
administration of ivermectin at school and community level before two months of actual data collection. In these districts the majority of communities are pastoralists, moving from one area to another to feed their domestic animals.

\section{Study Design and Period}

A community-based cross-sectional study was employed from April 1 to 30, 2019 G.C.

\section{Sample Size and Sampling Technique}

The name of kebeles (the smallest administrative unit in Ethiopia) involved and the number of segments selected for the survey were predetermined using a lottery method prior to the field work. From each district a total of 30 segments were randomly selected. Moreover, the number of households involved in the survey from each segment was determined before the actual data collection. In the field, data collectors visited the kebeles to assess the total number of households and population. Then, the data collectors divided the total households in each kebele by 50 to determine the number of segments.

From the total segments, the number of segments included in the survey was determined by a coverage survey builder tool and the numbers of segments surveyed were randomly selected. From each selected segment, at least 16 randomly selected households were included in the survey.

\section{Data Collection}

A semi-structured data collection tool was first drafted and evaluated by experts from $\mathrm{FMoH}$ and Ethiopia Public Health Institute (EPHI). It was then pre-tested on a small number of participants outside the study area. Health professionals who did not participate in the MDA campaign were intensively trained on the tool and how to approach study participants. From each selected household all members aged five years and above were interviewed since the treatment was given to individuals aged 5 years and above. If there was no member of the survey population in the selected household after repeated visits, or if the entire household was absent and not expected to return later in the day, the survey team proceeded to the next selected household.

\section{Variables}

\section{Dependent variables}

- Swallowed ivermectin (yes or no)
- Offered ivermectin (yes or no)
Independent Variables
- Age
- Sex
- Heard about mass drug administration
- Name of district
- School attendance

\section{Data Processing and Analysis}

Microsoft Excel was used to compute and clean the collected data, which was further analyzed by STATA version 14. In this survey treatment coverage is defined as proportion of study population who swallowed the drug. Data were disaggregated according to ivermectin offered and ivermectin swallowed, along with other sociodemographic variables. Chi-square test $\left(\mathrm{X}^{2}\right)$ test was used to check the difference among different variables. Finally the result was presented by frequency, graph and text.

\section{Data Quality Control}

Three days of intensive training was given for data collectors and supervisors on the management of the data collection tool by an expert from EPHI. In each district one supervisor and four data collectors were recruited. Data collectors and supervisors were instructors from Arba Minch University, College of Medicine and Health Sciences (CMHS) who had master's degree and above. In addition, they had previous experience on research and were certified on data collection processes. Data collectors took GPS of each house during the house-to-house survey using a mobile device. Daily supervision and checkup of collected data was made by the team members.

\section{Ethical Considerations}

This study was conducted in accordance with the Declaration of Helsinki. This study was ethically approved by Institute Research Board of Arba Minch University, CMHS, Ethiopia. Before data collection, written informed consent was obtained from each household head and/or participant after informing them of the purpose of the survey. Information for children ( $<10$ years) was collected from the children themselves or their caretakers. The interview was conducted only if he or she agreed to sign the digital consent form on the data collection devices. Confidentiality of the data was well ensured. 
Table I Ivermectin Offering Disaggregated by Socio-Demographic Characteristic of Study Participants, Ethiopia 2019

\begin{tabular}{|c|c|c|c|c|c|c|}
\hline \multirow[t]{2}{*}{ Characteristics } & \multirow[t]{2}{*}{ Category } & \multirow[t]{2}{*}{ Study Population } & \multicolumn{2}{|c|}{ IVM Offered } & \multirow[t]{2}{*}{$\mathbf{x}^{2}$} & \multirow[t]{2}{*}{$P$-value } \\
\hline & & & Yes N (\%) & No $N(\%)$ & & \\
\hline \multirow[t]{2}{*}{ District } & Wombera & $|68|$ & I539 (91.4) & I 42 (8.5) & \multirow[t]{2}{*}{70.467} & \multirow[t]{2}{*}{$P<0.00 I^{*}$} \\
\hline & Itang & 2077 & $1705(81.9)$ & $372(17.9)$ & & \\
\hline \multirow[t]{2}{*}{ Gender } & Male & 1849 & $1610(86.8)$ & $239(12.9)$ & \multirow[t]{2}{*}{1.741} & \multirow[t]{2}{*}{$P=0.187$} \\
\hline & Female & 1909 & $1634(85.6)$ & $275(14.4)$ & & \\
\hline \multirow[t]{2}{*}{ Age category (years) } & $5-14$ & 1124 & $978(86.6)$ & $146(12.9)$ & \multirow[t]{2}{*}{0.64} & \multirow[t]{2}{*}{$P=0.42$} \\
\hline & $\geq 15$ & 2634 & $2266(85.9)$ & $368(14)$ & & \\
\hline \multirow[t]{2}{*}{ SAC attendance } & Yes & 955 & $867(90.6)$ & $88(9.2)$ & \multirow[t]{2}{*}{77.29} & \multirow[t]{2}{*}{$P<0.00 I^{*}$} \\
\hline & No & 169 & III (64.53) & $58(33.73)$ & & \\
\hline
\end{tabular}

Note: *Variables with $P$-value $<0.05$.

\section{Results}

\section{Socio-Demographic Characteristics}

Two districts were involved in the coverage validation survey of onchocerciasis from Gambella and BenishangulGumuz regional states of Ethiopia. A total of 3822 individuals were targeted for the survey in the districts, 2125 from Itang special and 1697 from Wombera district. Out of these, 3765 (98.5\%) were approached for the interview. The remaining $57(1.5 \%)$ were excluded from analysis since they had missing values like offering and swallowing status of the participants. Among interviewed individuals seven did not know whether they had been offered the drug. From visited individuals, 3244/3765 (86.2\%) reported that they were offered onchocerciasis treatment. Of those who reported that the treatment was offered to them, $99.7 \%$ reported that they swallowed the drug.
The proportions of individuals who were offered ivermectin in Wombera (Benishangul-Gumuz) and Itang special districts (Gambella) were $91.4 \%$ and $81.9 \%$, respectively. Individuals who were living in Wombera district had more chance to be offered the treatment than Itang special $\left(\mathrm{X}^{2}=70.467, P<0.001\right)$. Children who attended school had more chance to be offered IVM than non-school attending children $\left(\mathrm{X}^{2}=77.29, P<0.001\right)$ (Table 1$)$.

\section{Treatment Coverage of Ivermectin}

The overall treatment coverage of ivermectin was $85.9 \%$ $(3235 / 3765)(95 \%$ CI: $84.8 \%, 87 \%)$. The treatment coverage of IVM in Wombera and Itang special districts were 91\% (1537/1683) $(95 \%$ CI: $89.9 \%, 92.6 \%)$ and $81.5 \%$ $(1698 / 2082) \quad(95 \%$ CI: $79.8 \%, 83.2 \%)$, respectively (Table 2).

Table 2 Treatment Coverage of Ivermectin Against Onchocerciasis in Ethiopia, 2019

\begin{tabular}{|c|c|c|c|c|}
\hline Characteristics & Category & Frequency (Study Population) & Treated with IVM & Treatment Coverage (\%) \\
\hline \multirow[t]{3}{*}{ Districts } & Wombera & 1683 & 1537 & 91.3 \\
\hline & Itang special & 2082 & 1698 & 81.5 \\
\hline & Overall & 3765 & 3235 & 85.9 \\
\hline \multirow[t]{2}{*}{ Gender } & Male & 1855 & 1606 & 86.6 \\
\hline & Female & 1910 & 1629 & 85.3 \\
\hline \multirow[t]{2}{*}{ Age category (years) } & $5-14$ & 1129 & 973 & 86.2 \\
\hline & $\geq 15$ & 2636 & 2262 & 85.8 \\
\hline \multirow[t]{2}{*}{ School attendance } & Yes & 957 & 863 & 90.2 \\
\hline & No & 172 & 110 & 64 \\
\hline
\end{tabular}


Table 3 Ivermectin Swallowing Status Among Individuals Who Were Offered Treatment, Disaggregated with Other Variables, Ethiopia, 2019

\begin{tabular}{|c|c|c|c|c|c|c|c|}
\hline \multirow[t]{2}{*}{ Characteristics } & \multirow[t]{2}{*}{ Category } & \multirow[t]{2}{*}{ IVM Offered } & \multicolumn{3}{|c|}{ Swallowed IVM } & \multirow[t]{2}{*}{$\mathbf{x}^{2}$} & \multirow[t]{2}{*}{$P$-value } \\
\hline & & & Yes (\%) & No (\%) & Unknown (\%) & & \\
\hline \multirow[t]{2}{*}{ District } & Wombera & 1539 & 1537 (99.9) & I (0.06) & I (0.06) & \multirow[t]{2}{*}{5.031} & \multirow[t]{2}{*}{$0.08 I$} \\
\hline & Itang & 1705 & 1698 (99.6) & $7(0.4)$ & 0 & & \\
\hline \multirow[t]{2}{*}{ Gender } & Male & 1610 & 1606 (99.7) & $3(0.2)$ & $\mathrm{I}(0 . \mathrm{I})$ & \multirow[t]{2}{*}{1.486} & \multirow[t]{2}{*}{0.476} \\
\hline & Female & 1634 & 1629 (99.7) & $5(0.3)$ & 0 & & \\
\hline \multirow[t]{2}{*}{ Age in years } & $5-14$ & 978 & $973(99.5)$ & $4(0.4)$ & $\mathrm{I}(0.1)$ & \multirow[t]{2}{*}{3.822} & \multirow[t]{2}{*}{0.148} \\
\hline & $\geq 15$ & 2266 & $2262(99.8)$ & $4(0.2)$ & 0 & & \\
\hline \multirow[t]{2}{*}{ SAC attendance } & Yes & 867 & $863(99.5)$ & $4(0.5)$ & 0 & \multirow[t]{2}{*}{30.581} & \multirow[t]{2}{*}{0.001} \\
\hline & No & 111 & II 0 (99.I) & 0 & I (0.9) & & \\
\hline
\end{tabular}

The treatment coverage of offered IVM was $99.9 \%$ and $99.6 \%$ in Wombera and Itang special districts, respectively. In this study, equal proportion of males and females were treated, 99.7\%. Only school attendance was significantly associated with swallowing status of the participants; those children who attended school had more chance of swallowing IVM than others $\left(\mathrm{X}^{2}=30.581\right.$, $P=0.001$ ) (Table 3).

\section{Reasons for Not Being Offered Onchocerciasis Treatment}

A total of 514/3765 (13.7\%) of individuals were not offered onchocerciasis treatment due to different reasons. The main reported reasons for not being offered IVM were "being absent" (40.86\%) during MDA campaign and "did not know about MDA" (25.29\%) (Figure 1).

Among offered individuals, very few $(8 ; 0.2 \%)$ did not swallow the drug. The reasons for respondents not swallowing ivermectin were "no information", "bad taste", "not sick", "not around at the time of deworming" and "nobody came".

\section{Discussion}

As annual and semi-annual mass drug administration with ivermectin is the main strategy for elimination of onchocerciasis in Ethiopia, validation coverage survey is important to improve the national program. Although we had routine coverage reports in the health system, these reports are subject to errors and their accuracy is doubtful. The desired therapeutic coverage for ivermectin is at least $80 \%$ of the eligible population, as per the WHO recommendation. ${ }^{15}$

However, our coverage survey showed an overall treatment coverage of ivermectin of $85.9 \%$. This is in line with the reported coverage of similar year (Figure 2), and it is above the minimum recommended threshold $(80 \%) .{ }^{16}$ The treatment coverage in the current survey is higher than the 2017 and 2016 national coverage of ivermectin, which were $81 \%$ and $80.1 \%$, respectively. ${ }^{16}$ This might be explained by the fact that treatment was given more than four times in the area and thus people became familiar with and more aware of the importance of the treatment.

A total of 3244 participants (86.2\%) reported that they were offered the treatment. Almost all individuals who were offered treatment swallowed the drug. The possible explanation for this finding might be the effort of drug distributers to address the whole community. In contrast, MDA for onchocerciasis treatment in Nigeria Edo state in 2016 reported that most of the respondents were not offered IVM. ${ }^{17}$

According to the study, individuals who were living in Wombera district had more chance to be offered ivermectin than Itang special. This might be due to variation in commitment between the districts.

This survey found that school attendance was significantly associated with offering of onchocerciasis treatment $\left(\mathrm{X}^{2}=87, P<0.001\right)$. The likely reason behind this might be the fact that school-aged children could be well informed about the importance of the treatment by their respective teachers at school, and even if the treatment was given at community level those children who did not attend school went to keep domestic animals. 


\section{Percent $\quad$ Frequency}

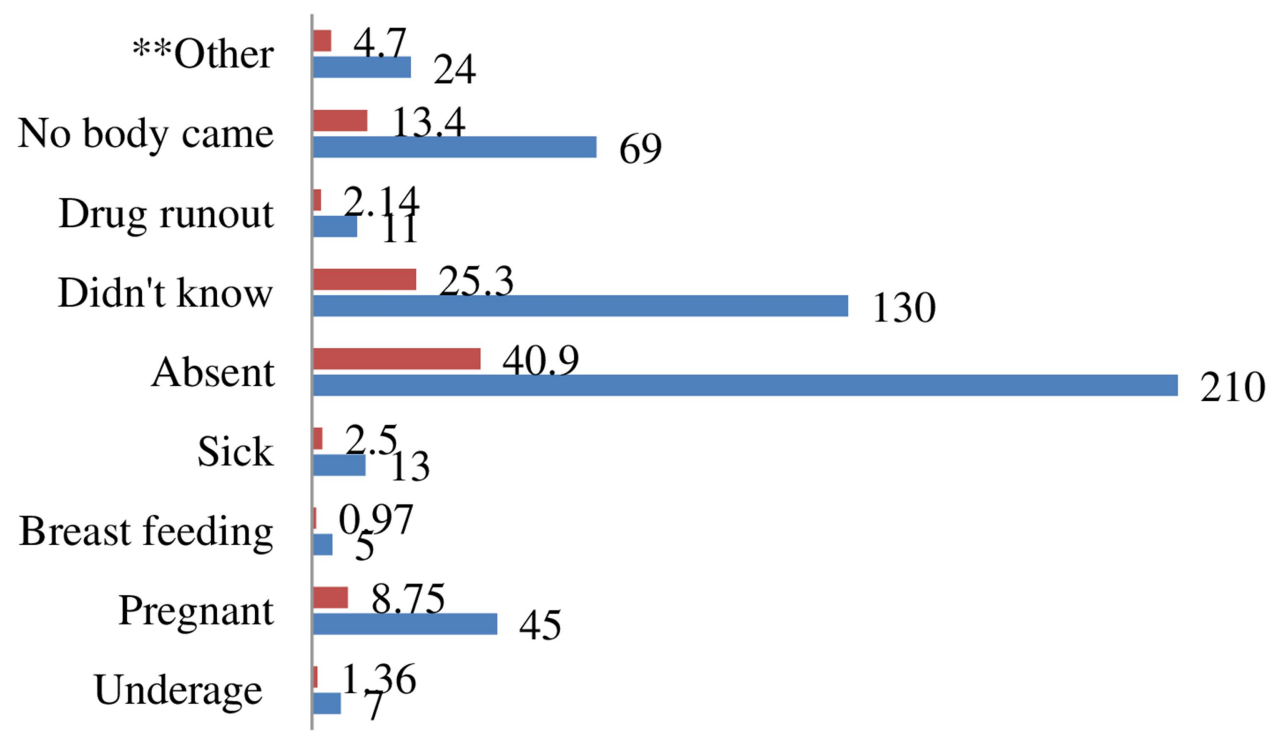

Figure I Reported reasons of participants for not being offered treatment for onchocerciasis during MDA, 2019 ( $N=5$ I4). (**Others include "did not like drugs", "not given for adults", "do not attend schools", "forgot about the deworming", "fear of side effects" and "unable to move").

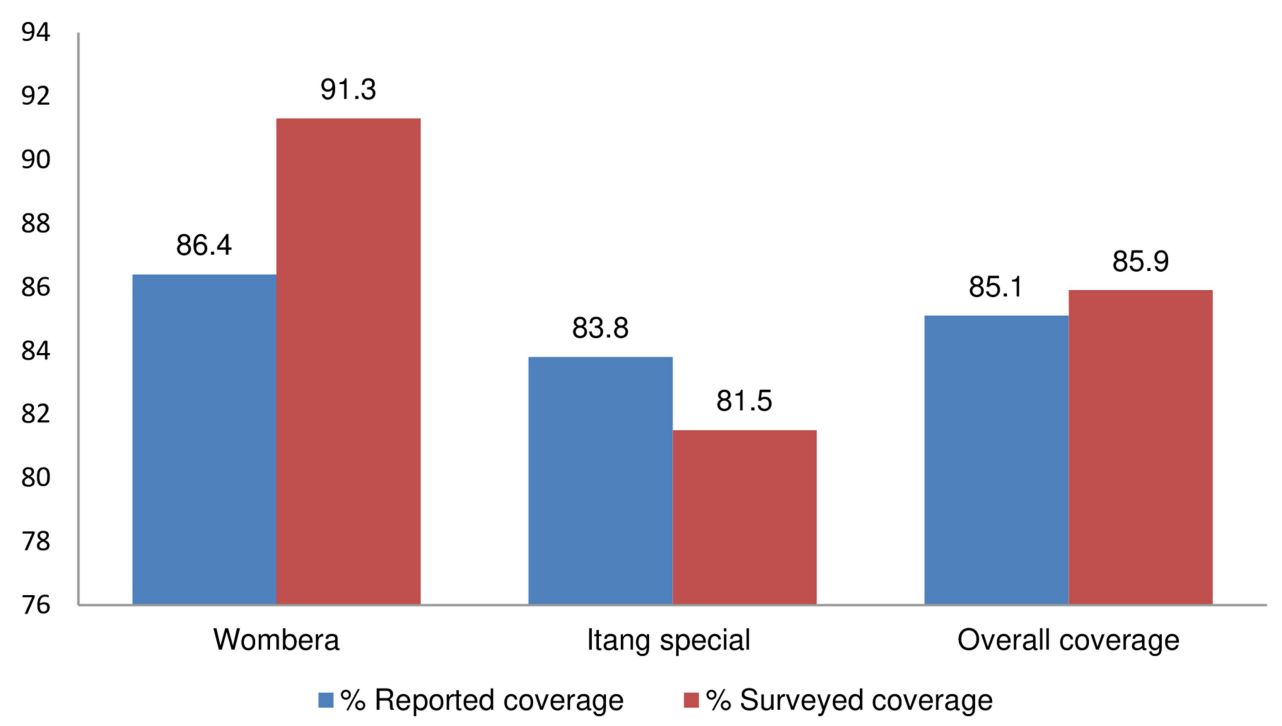

Figure 2 Comparison between reported and surveyed PC coverage against onchocerciasis in Wombera and Itang special districts in Ethiopia, 2019.

The strength of our study includes the following: Firstly there was high response rate of study population. Secondly, well trained and experienced university instructors collected the data for this survey. Limitations of this study include: Firstly, we used purposive sampling technique to select regions; in this case it is difficult to generalize the findings to national level. Secondly, there may be recall and social desirability bias.

\section{Conclusion and Recommendation}

In conclusion, the treatment coverage of onchocerciasis in this survey was higher than the minimum recommended national therapeutic coverage for onchocerciasis. Treatment coverage in Wombera was higher than Itang special district. Treatment offered was significantly higher in Wombera than in Itang special district. In addition, attending school was associated with a higher chance of being offered and swallowing the drug. Although the 
overall treatment coverage is above the WHO minimum recommendation, substantial numbers of individuals were not offered the treatment: 514 eligible individuals were not offered onchocerciasis treatment due to different reasons. The main reported reason was "being absent during the campaign period". It would be better to improve the distribution, to make sure that all individuals get offered treatment. In areas where people live a pastoralist lifestyle a mobile community-based campaign would be appropriate towards the treatment before mass drug campaigns. Furthermore, emphasis should be given to communitybased intervention to better increase the coverage to reach all segments of the population.

\section{Abbreviation}

AIDS, acquired immune deficiency syndrome; APOC, Africa Program for Onchocerciasis Control; BG, Benishangul-Gumuz; CDTI, community directed treatment with ivermectin; DALYS, Disability Adjusted Life Years; EPHI, Ethiopian Public Health Institute; $\mathrm{FMoH}$, Federal Ministry of Health; IVM, ivermectin; MDA, mass drug administration; NGO, non-governmental organization; NTDs, neglected tropical diseases; OCP, Onchocerciasis Control Program; PC, preventive chemotherapy; REMO, Rapid Epidemiological Mapping for Onchocerciasis; SAC, school age children; SCI, schistosomiasis control initiative; SDG, sustainable development goal; SNNP, Southern Nations Nationalities People; WHO, World Health Organization.

\section{Data Sharing Statement}

The datasets during and/or analyzed during the current study are available from the corresponding author on reasonable request.

\section{Acknowledgments}

We would like to acknowledge $\mathrm{FMoH}$, evidence action and schistosomiasis control initiative for their technical and financial support to undertake this coverage validation survey. Our gratitude also goes to NTD focal person in the districts involved in the study for providing information about the kebeles and the mass drug administration. We also thank guiders directing the households in each segment, research assistants who administered the questionnaires and individual participants involved in the study.

\section{Author Contributions}

All authors made a significant contribution to the work reported, whether that is in the conception, study design, execution, acquisition of data, analysis and interpretation, or in all these areas; took part in drafting, revising or critically reviewing the article; gave final approval of the version to be published; have agreed on the journal to which the article has been submitted; and agree to be accountable for all aspects of the work.

\section{Funding}

Source of support: The fund for collection of the data including costs for vehicle rent, allowance for data collectors and other contingencies were covered by FMOH.

\section{Disclosure}

The authors declare that they have no competing interests.

\section{References}

1. Tekle AH, Zouré HG, Noma M, et al. Progress towards onchocerciasis elimination in the participating countries of the African Programme for Onchocerciasis Control: epidemiological evaluation results. Infect Dis Poverty. 2016;5(1):66. doi:10.1186/s40249-0160160-7

2. World Health Organization. Onchocerciasis key fact sheet; 2019. Available from: https://www.who.int/news-room/fact-sheets/detail/ onchocerciasis. Accessed December 18, 2020.

3. Little MP, Breitling LP, Basáñez MG, Alley ES, Boatin BA. Association between microfilarial load and excess mortality in onchocerciasis: an epidemiological study. Lancet. 2004;363 (9420):1514-1521. doi:10.1016/S0140-6736(04)16151-5

4. Murdoch ME, Hay RJ, Mackenzie CD, et al. A clinical classification and grading system of the cutaneous changes in onchocerciasis. $\mathrm{Br}$ J Dermatology. 1993;129(3):260-269. doi:10.1111/j.1365-2133.1993. tb11844.x

5. Murray CJ, Barber RM, Foreman KJ, et al. Global, regional, and national disability-adjusted life years (DALYs) for 306 diseases and injuries and healthy life expectancy (HALE) for 188 countries, 1990-2013: quantifying the epidemiological transition. Lancet. 2015;386(10009):2145-2191. doi:10.1016/S0140-6736(15)61340-X

6. WHO. Regional Strategic Plan for NTDs in the African Region; 2020.

7. WHO/APOC. Status of Onchocerciasis in APOC Countries; 2013.

8. Crump A, Morel CM, Omura S. The onchocerciasis chronicle: from the beginning to the end? Trends Parasitol. 2012;28(7):280-288. doi:10.1016/j.pt.2012.04.005

9. World Health Organization. Report of the External Mid-Term Evaluation of the African Programme for Onchocerciasis Control. African Programme for Onchocerciasis Control; 2010.

10. World Health Organization. Guidelines for Stopping Mass Drug Administration and Verifying Elimination of Human Onchocerciasis: Criteria and Procedures. World Health Organization; 2016.

11. Dana D, Debalke S, Mekonnen Z, et al. A community-based cross-sectional study of the epidemiology of onchocerciasis in unmapped villages for community directed treatment with ivermectin in Jimma Zone, southwestern Ethiopia. BMC Public Health. 2015;15 (1):595. doi:10.1186/s12889-015-1888-x 
12. Dori GU, Belay T, Belete H, Panicker KN, Hailu A. Parasitological and clinico-epidemiological features of onchocerciasis in West Wellega, Ethiopia. J Parasitic Dis. 2012;36(1):10-18. doi:10.1007/ s12639-011-0063-2

13. HR PM T, Muñoz B, Greene BM, Greene B. Impact of MDA of onchocerciasis with ivermectin on the transmission of infection. Science. 1990;250(4977):116-118. doi:10.1126/science. 2218502

14. UN. Transforming our world, the 2030 agenda for sustainable development; 2020. Available from: sustainabledevelopment.un.org. Accessed December 18, 2020.
15. Meribo K, Kebede B, Feleke SM, et al. Review of Ethiopian onchocerciasis elimination programme. Ethiop Med J. 2017;55(Suppl 1):55.

16. WHO. Neglected Tropical Diseases-Onchocericiasis Data; 2019.

17. Griswold E, Eigege A, Ityonzughul C, et al. Evaluation of treatment coverage and enhanced mass drug administration for onchocerciasis and lymphatic filariasis in five local government areas treating twice per year in Edo state, Nigeria. Am J Trop Med Hyg. 2018;99 (2):396-403. doi:10.4269/ajtmh.17-1004

\section{Publish your work in this journal}

The Journal of Multidisciplinary Healthcare is an international, peerreviewed open-access journal that aims to represent and publish research in healthcare areas delivered by practitioners of different disciplines. This includes studies and reviews conducted by multidisciplinary teams as well as research which evaluates the results or conduct of such teams or healthcare processes in general. The journal covers a very wide range of areas and welcomes submissions from practitioners at all levels, from all over the world. The manuscript management system is completely online and includes a very quick and fair peer-review system. Visit http://www.dovepress.com/testimonials. php to read real quotes from published authors. 